\title{
Correlation Between Point Shear Wave Elastography and Liver Function Tests as A Predictor of Liver Fibrosis in Patients with Nonalcoholic Fatty Liver Disease
}

\author{
SHAHLA MOHAMMED SAEED RASUL ${ }^{1 *}$, ALI KHALAF SALIM ${ }^{2}$, HIWA ABUBAKR HUSSEIN ${ }^{3}$ \\ ${ }_{1,3}^{3}$ College of Medicine, Sulaymaniyah University, Sulaymaniyah, Iraq \\ ${ }^{2}$ Kirkuk Oncology and Hematology Center, Kirkuk Health Directorate, Kirkuk, Iraq \\ ${ }^{*}$ Corresponding Author, email: barzinjishahla@gmail.com
}

\begin{abstract}
Background: Nowadays, generating shear waves and simulation of the liver tissue is done using point shearwave elastographic ( $p S W E$ ) techniques which uess acoustic radiation force impulse (ARFI).

Objective: This study aimed to evaluate the correlation between pSWE and liver function tests (LFTs) to predict liver fibrosis in patients with non-alcoholic fatty liver disease (NAFLD).

Materials and methods: It was a cross sectional study conducted in an Ultrasound Clinic in Suleymaniya city. The duration of the study was from $1^{\text {st }}$ of November, 2018 to $30^{\text {th }}$ of June, 2019 which conducted on 50 NAFLD patients. After confirming NAFLD diagnosis, the patients were referred to Ultrasound Clinic to go under pSWE test.

Results: The data showed that the mean PSWE of NAFLD patient was $4.12 \pm 0.87 \mathrm{Kpa}$; and $18 \%$ of them had high PSWE (> 4.6). Elastography fibrosis score was distributed to F0 (82\%), F1 (6\%), F2 (8\%) and F3 (4\%). There was a significant association between high APRI and high Aspartate Aminotransferase/Alanine Aminotransferase(AST/ALT) ratio $(p=0.04)$. There was also a highly significant association between elastography fibrosis score and APRI fibrosis score among NAFLD patients $(p<0.001)$.

Conclusion: This study showed that the pSWE is a valuable noninvasive diagnostic technique for predicting liver fibrosis among NAFLD patients and there is significant correlation between APRI and pSWE scores.

Keywords: Non-alcoholic fatty liver disease, Point shears wave elastography, Liver fibrosis.
\end{abstract}

\section{INTRODUCTION}

Liver fibrosis is a progressive disease, so its early diagnosis leading to early clinical intervention that may slow down or stop progression to cirrhosis [1]. Many chronic liver diseases end with liver fibrosis. One of them Non-alcoholic fatty liver disease (NAFLD) is. NAFLD is associated with overweight and insulin resistance. Chronic metabolic conditions such as diabetes and degenerative conditions like atherosclerosis also may lead to pathological changes in the biochemical profile of the liver that lead to liver fibrosis [2]. NAFLD is a wide term covering form simple non-alcoholic fatty liver (NAFL), in which there is pure hepatosteatosis only, mild inflammatory process, and non-alcoholic steatohepatitis (NASH). The existence of these three histopathological features, i.e., steatosis, inflammation, and ballooning (swollen hepatocytes), is required for $\mathrm{NASH}$, which also covers the most progressive forms of NAFLD, fibrosis, cirrhosis, and HCC [3].

Previous studies have introduced liver biopsy as one of the most appropriate diagnostic methods of NAFLD and the staging of liver fibrosis. Anyway, in spite of its effectiveness, it could cause several problems which include its high cost, available risk of complications, sampling error, and inter-, and intra-observer variation. Therefore, some other simple methods with a lower risk of invasiveness have been explored for the assessment of liver fibrosis [4].

Aspartate Aminotransferase Platelet Ratio Index (APRI) is a laboratory marker that has been shown to have some value in staging liver fibrosis but is lower to liver biopsy [5]. As diabetes, obesity, old age, platelet count $\leq$ $200 \times 109 / \mathrm{L}$, and Aspartate Aminotransferase/Alanine Aminotransferase $(A S T / A L T)$ ratio $\geq 0.8$ are risk factors of advanced fibrosis ( $\geq F 3$ ) in patients with NAFLD, there are many scoring systems found to predict fibrosis, mostly based on these variables. Of these, APRI index \& AST/ALT ratio4, The AST to ALT ratio is a useful index for distinguishing nonalcoholic steatohepatitis from alcoholic liver disease [6], The APRI has acceptable accuracy for the assessment of liver fibrosis in patients with Chronic Hepatitis $\mathrm{C}(\mathrm{CHC})$ and NAFLD, but not in those with Chronic Hepatitis B (CHB) [7].

Point shear-wave elastographic (pSWE) techniques are ultrasound-based techniques that using the radiation force from a focused ultrasound beam creates shear waves inside the liver. The velocity of the shear wave and its propagation is measured and monitored by an ultrasound machine. Assuming that the behavior of tissue is very simple, the velocity of the shear wave is determined to be related to the stiffness of tissue through Young's modulus. To measure the stiffness of tissue the kilopascal $(\mathrm{kPa})$ is used as its unit [8]. The application of shear wave elastography techniques has been reported to be effective in conventional real-time ultrasound systems, also their accuracy in the assessment of liver fibrosis has been proved through several studies [14-22]. In comparison with transient elastography (TE), these techniques have the advantage of B-mode image guidance. In this regard, they provide the possibility of choosing the best acoustic window for the users to perform an appropriate examination in realtime [9].

ARFI imaging is a model for evaluation of mechanical features of the tissue, without manual compression, by measuring the shear wave speed induced by acoustic radiation and propagating in the tissue. This quantitative technique gives a single uni-dimensional measurement of 
tissue elasticity like the FibroScan, although the measurement area can be positioned on a two-dimensional $B$ mode image [10].

Nowadays non-invasive techniques are developed for measuring liver fibrosis mainly as adequate alternatives to liver biopsy that have attracted the attention of many researchers. Among them, transient elastography (TE) is being used increasingly for the assessment of liver fibrosis [11]. On the other hand, two-dimensional shear wave elastography is becoming an interesting non-invasive diagnostic tool particularly useful for people with obesity. The objective of this study is to clarify the availability of any association between pSWE and liver function tests (LFTs) for the prediction of liver fibrosis in NAFLD patients [10].

\section{MATERIAL AND METHODS}

Sampling: This is a cross-sectional study conducted in an Ultrasound Clinic in Sulaymaniyah city. The duration of the study was from 1st of November, 2018 to 30th of June, 2019. All NAFLD patients underwent PSWE examination and liver function tests and some other laboratory investigations (illustrated in the questionnaire). Patients ranged in age from 18 to 70 years. Patients with bright echogenic liver on ultrasound scanning (increased liver/kidney echogenicity) were included in the study. While exclusion criteria were alcoholic patients, also patients with viral hepatitis, autoimmune hepatitis, Wilson's disease, hemochromatosis, and patients who refused to participate. A sample of 50 NAFLD patients was selected after eligibility to inclusion and exclusion criteria.

Data collection: The process of collection of data was carried out by the researcher by doing direct interviews with selected patients and filling in a prepared questionnaire. The questionnaire was designed by a gastroenterologist (experience of 5 years), researcher, and supervisor (has experience of 1.5 years in pSWE). After taking full history and examination of NALFD patients and calculating their body mass index (BMI), mean BMI was analyzed according to international BMI category as shown below:

\begin{tabular}{|l|l|}
\hline \multicolumn{2}{|l|}{ Table 1. International BMI Classification } \\
\hline Underweight & $<18.5 \mathrm{~kg} / \mathrm{m}^{2}$ \\
\hline Normal & $18.5 \mathrm{~kg} / \mathrm{m}^{2}-25 \mathrm{~kg} / \mathrm{m}^{2}$ \\
\hline Overweight & $\left(25 \mathrm{~kg} / \mathrm{m}^{2}-30 \mathrm{~kg} / \mathrm{m}^{2}\right.$ \\
\hline Obesity & $\geq 30 \mathrm{~kg} / \mathrm{m}^{2}$ as obesity ${ }^{16}$ \\
\hline
\end{tabular}

All the patients were sent for the laboratory investigations as described in the questioner. AST/ALT tests were carried out for all the patients, mean AST/ALT ratios of each patient were analyzed based on three groups of no fibrosis (0.7), mild fibrosis (0.9), and cirrhosis (1.4). The APRI score was calculated using a formula APRI = [(AST level/ULN of AST)/platelet counts $\left.\left(10^{9} / \mathrm{L}\right)\right] \times 100$. Moreover, a score of $<0.5$ was graded as F0, $0.5-1.5$ as F1- F3 and >1.5 as F4 [1]. The patients then were referred to perform pSWE assessment using the ultrasound machine of Philips Affiniti 70 with EPQ software (release 3.0.3).

The pSWE was performed for the patients after being fasting for at least 4 hours. Then an ultrasound-based pSWE exam was done after selecting the best acoustic window. The operator was informed that the region of interest $(\mathrm{ROI})$ is placed in an area of the liver perpendicular to the liver capsule and should take care not to include large vasculature or biliary structures. The intercostal imaging approach targets segments 7 or 8 of the liver. At least 10 validated measurements taken while the patient suspends respiration automatic median value generated by the ultrasound software EPQ (taking into consideration $\mathrm{IQR} /$ median ratio is $<30 \%$ ). The patients were divided into five groups, and the interpretation of liver fibrosis by point shear wave elastography was done according to Philips vendor cutoff value in $\mathrm{kPa}$ :

\begin{tabular}{|l|l|l|}
\hline \multicolumn{3}{|l|}{ Table 2. Classification of liver fibrosis } \\
\hline 1 & No fibrosis & $\mathrm{F} 0<4.6 \mathrm{KPa}$ \\
\hline 2 & Mild fibrosis & $\mathrm{F} 14.6-5.6 \mathrm{KPa}$ \\
\hline 3 & Moderate fibrosis & $\mathrm{F} 25.7-7 \mathrm{KPa}$ \\
\hline 4 & Marked fibrosis & $\mathrm{F} 37.1-12 \mathrm{KPa}$ \\
\hline 5 & Cirrhosis $^{*}$ & $\mathrm{~F} 4>12 \mathrm{KPa}$ \\
\hline${ }^{*}$ was used to establish the elastography grade1 \\
\hline
\end{tabular}

\section{Ethical considerations:}

- An approval was taken from Suleymaniya General Directorate of Health

- An oral informed consent was taken from patients.

The patients' data were analyzed using Microsoft excel software program and Statistical Package for Social Sciences (SPSS) version 23. The results of analysis were arranged in scales variables (means \& standard deviation) and in categorical variables. Chi square test was used for conducting a comparison analysis between categorical data (Fishers exact test applied when expected variable was less than $20 \%$ of total). The level of significance ( $p$ value) was set as $\leq 0.05$.

\section{RESULTS}

In the present study, 50 patients with NAFLD disease were included with a mean age of $44.5 \pm 10$ years. $28 \%$ of them were less than 40 years, $38 \%$ of them were in the age group $40-49$ years, $24 \%$ of them were in the age group 50 59 years and $10 \%$ of them were 60 years or older. Half of the patients were male and the other half were female. The mean weight of NAFLD patients was $89.5 \pm 19.1 \mathrm{Kg}$ and mean height was $1.69 \pm 0.08 \mathrm{~m}$, while the mean $\mathrm{BMl}$ was $31.6 \pm 6 \mathrm{Kg} / \mathrm{m} 2$. $16 \%$ of NAFLD patients had a normal BMI, $26 \%$ of them were overweight and $58 \%$ of them were obese (Table 3 ).

\begin{tabular}{l}
\begin{tabular}{|l|l|l|}
\hline \multicolumn{3}{|l|}{ Table 3. Demographic characteristics of NAFLD patients. } \\
\hline Variable & No. & $\%$ \\
\hline Age mean \pm SD $(44.5 \pm 10$ years $)$ & 28.0 \\
\hline$<40$ years & 14 & 38.0 \\
\hline $40-49$ years & 19 & 24.0 \\
\hline $50-59$ years & 12 & 10.0 \\
\hline$\geq 60$ years & 5 & 100.0 \\
\hline Total & 50 & 50.0 \\
\hline Gender & \multicolumn{2}{|l|}{} \\
\hline Male & 25 & 50.0 \\
\hline Female & 25 & 100.0 \\
\hline Total & 50 & \multicolumn{2}{|l}{} \\
\hline Weight & Mean $\pm \mathrm{SD}(89.5 \pm 19.1 \mathrm{Kg})$ & 16.0 \\
\hline Height & Mean $\pm \mathrm{SD}(1.69 \pm 0.08 \mathrm{~m})$ & 26.0 \\
\hline BMI & Mean $\pm \mathrm{SD}\left(31.6 \pm 6 \mathrm{Kg} / \mathrm{m}^{2}\right)$ & 58.0 \\
\hline Normal & 8 & 100.0 \\
\hline Overweight & 13 & \\
\hline Obese & 29 & \\
\hline Total & 50 &
\end{tabular} \\
\hline
\end{tabular}


Mean ALT of NAFLD patients was $73.4 \pm 59.3 \mathrm{U} / \mathrm{L}$; and $50 \%$ of them had high ALT. Moreover, mean AST of NAFLD patients was $58.5 \pm 89.9 \mathrm{U} / \mathrm{L}$; and $64 \%$ of them had high AST. Upper limit of normal AST was $37.2 \pm 2.6 \mathrm{U} / \mathrm{L}$. The mean of platelets count of NAFLD patients was $228.4 \pm 50.6 \times 109 / L$, and only of the patients had low platelets count (Table 4).

\begin{tabular}{|l|l|l|l|}
\hline \multicolumn{3}{|c|}{ Table 4: laboratory investigations of NAFLD patients. } \\
\hline Variable & No. & Mean \pm SD $(73.4 \pm 59.3 \mathrm{U} / \mathrm{L})$ \\
\hline \multicolumn{3}{|c|}{ ALT } & 50.0 \\
\hline Normal & 25 & Mean \pm SD $(58.5 \pm 89.9 \mathrm{U} / \mathrm{L})$ \\
\hline High & 25 & 100.0 & 36.0 \\
\hline Total & 50 & & 64.0 \\
\hline \multicolumn{3}{|c|}{ AST } & 100.0 \\
\hline Normal & 18 & Mean \pm SD $(37.2 \pm 2.6 \mathrm{U} / \mathrm{L})$ \\
\hline High & 32 & Mean \pm SD $\left(228.4 \pm 50.6 \times 10^{9} / \mathrm{L}\right)$ \\
\hline Total & 50 & 98.0 \\
\hline \multicolumn{2}{|c|}{ Ulatelets count } & 2.0 \\
\hline Normal & 49 & 100.0 \\
\hline Low & 1 &
\end{tabular}

Mean PSWE of NAFLD patients was $4.12 \pm 0.87 \mathrm{Kpa}$; while $18 \%$ of them were detected with high PSWE. The distribution of elastography fibrosis score was as follow F0 (82\%), F1 (6\%), F2 (8\%) and F3 (4\%) (Table 5).

\begin{tabular}{|c|c|c|}
\hline Variable & No. & \multirow{2}{*}{$\frac{\%}{\text { Mean } \pm \text { SD }(4.12 \pm 0.87 \mathrm{Kpa})}$} \\
\hline \multicolumn{2}{|c|}{ PSWE } & \\
\hline Normal & 41 & 82.0 \\
\hline High & 9 & 18.0 \\
\hline Total & 50 & 100.0 \\
\hline \multicolumn{3}{|c|}{ Elastography fibrosis score } \\
\hline $\mathrm{F} 0<4.6 \mathrm{KPa}$ & 41 & 82.0 \\
\hline $\mathrm{F} 1(4.6-5.6) \mathrm{KPa}$ & 3 & 6.0 \\
\hline $\mathrm{F} 2(5.7-7) \mathrm{KPa}$ & 4 & 8.0 \\
\hline $\mathrm{F} 3(7.1-12) \mathrm{KPa}$ & 2 & 4.0 \\
\hline $\mathrm{F} 4>12 \mathrm{KPa}$ & 0 & 0 \\
\hline Total & 50 & 100.0 \\
\hline
\end{tabular}

As could be seen from table 6 , there is a highly significant association between elastography fibrosis score and APRI fibrosis score of NAFLD patients $(p<0.001)$.

\begin{tabular}{|c|c|c|c|c|}
\hline Variable & F0 APRI & F1-3 APRI & F4 APRI & \multirow{5}{*}{$P<0.001^{*} \mathrm{~S}$} \\
\hline F0 pSWE & 22 & 19 & 0 & \\
\hline F1 pSWE & 0 & 3 & 0 & \\
\hline F2 pSWE & 2 & 2 & 0 & \\
\hline F3 pSWE & 0 & 0 & 2 & \\
\hline
\end{tabular}

It's while, there was no significant association between elastography fibrosis score and AST/ALT values of NAFLD patients $(p=0.5)$ (Table 7$)$.

\begin{tabular}{|c|c|c|c|c|}
\hline Variable & No fibrosis & Mild fibrosis & cirrhosis & $\mathrm{P}$ \\
\hline F0 pSWE & 17 & 24 & 0 & \multirow{4}{*}{ NS } \\
\hline F1 pSWE & 2 & 1 & 0 & \\
\hline F2 pSWE & 1 & 3 & 0 & \\
\hline F3 pSWE & 0 & 2 & 0 & \\
\hline
\end{tabular}

Only three NAFLD patients have liver biopsy before the laboratory investigations, and then pSWE done for them and the results of this laboratory test and pSWE result as shown in table 8.

\begin{tabular}{|l|l|l|l|}
\hline Table 8. pSWE, AST/ALT ratio, APRI score and histological results. \\
\hline Biopsy result & AST/ALT ratio & APRI score & pSWE \\
\hline F0 & 0.5 & 0.6 & 3.71 \\
\hline F0 & 0.5 & 0.3 & 3.39 \\
\hline F1 & 0.8 & 0.9 & 5.3 \\
\hline
\end{tabular}

\section{DISCUSSION}

Liver fibrosis is among the most common complications in majority of chronic liver diseases. The accurate staging of fibrosis is mandatory, especially for prognosis, disease management, treatment indication and long-term follow-up. PSWE is one of the non-invasive method in assessing liver fibrosis [12-17]. Our study showed that the patints within the age range of 40-49 years were presented with higher incidence of NAFLD with an equal incidence between male and female. A similar study by Shiobhan et al [18] revealed that the mean age of the NAFLD patients was 50 (range, 20-74 years) while $52.2 \%$ of the participants were male. It's while, Benedict et al [19], stated that the role of gender in the development of NAFLD has faced different conclusions in the literature. However, according to Lonardo et al [19] studies, NAFLD is more common among men and will increase in those who are at the age range of young to middle age and then it will be decreased notably after the age of 50-60 years.

In the present study, about $58 \%$ of participants were obese. This result is not in line with the data achieved from the study by Manopriya et al [20] who showed that NAFLD is more common in obese that may be due to the large sample size, racial and probably ethnic factors. In our study it was revealed that only $2 \%$ of participants have a low platelet count that is in contrast with the results achieved by Das S K et al [21] who demonstrated that platelet count will significantly decrease in patients with NAFLD disease. The main reason for this dissimilarity in our results may be our smaller smaple size and also the fact that more than fourfifths of our NAFLD patients were within (F0) category.

Honda et al [22] showed that obesity seems to have less influence on pSWE. The accuracy of pSWE could be affected by the presence of hepatic steatosis. It's while another study by Cassinotto et al [23] revealed that measuring liver stiffness by pSWE could not be reliable in about one-fifth of NAFLD patients.

The data from the present study demonstrated that in $40 \%$ of patients AST/ALT ratio was normal and in the rest of them the ratio of AST/ALT was high. This is in line with the result of a similar study carried out by Sorbi et al [6], who stated that abnormal AST to ALT ratio are suggestive of non-alcoholic steatohepatitis. Based on a study carried out by Yilmaz et al [24] AST to platelet ratio index shows an acceptable accuracy for the assessment of liver fibrosis in patients with NAFLD. These results are in line with the data achieved from the present study that showed $52 \%$ of participants have a high APRI score value.

The elastography fibrosis score in our study was distributed to F0 (82\%), F1 (6\%), F2 (8\%) and F3 (4\%) that was not similar to the results achieved by Masato Yoneda et al [22] who showed the categorization of NAFLD patients 
according to the elastography fibrosis score was $\mathrm{F} 0(25 \%)$, $\mathrm{F} 1(31 \%), \mathrm{F} 2(12 \%), \mathrm{F} 3(6 \%)$ and $\mathrm{F} 4(9 \%)$. This dissimilarity may be due to that they used another vender so there was a difference in their categorization. Moreover, each vender had its own cutoff values and the majority of our NAFLD patients were within $F(0)$ category (simple steatosis) where the fibrosis is not developed yet. The data from this study revealed that the is a high association between elastography fibrosis score and APRI fibrosis score of NAFLD patients. This finding is in line with those achieved by Benedict et al [19] who showed a significant correlation between pSWE and APRI score which emphasizing the important role of pSWE among NAFLD with abnormal APRI score.

A study by $\mathrm{Li}$ et al [25] revealed that AST/ALT ratio in $\mathrm{NASH}$ patients is normal that is in line with the data from our study which doesn't show a significant association between pSWE fibrosis score and AST/ALT ratio. The pSWE is a noninvasive tool for assessment of liver fibrosis in NAFLD [26]. In our study only three patients had LB in which pSWE grades are matching those of their LB grades that emphasizes the significant role of pSWE in assessment of liver fibrosis in NAFLD patients. The main limitations were the absence of liver biopsy, relatively small sample size, limited number of published articles using pSWE among NAFLD patients. Moreover, there was any standardized fibrosis score among different ultrasound vendors as each vendor has its own elastography fibrosis score.

\section{CONCLUSION}

The new point shear-wave elastography method of $p-S W E$ is a good tool for assessment of liver stiffness that shows a good correlation with transient elastography. This method is capable of providing good diagnostic performance in the prediction of hepatic fibrosis stages that doesn't provide a high rate of successful liver stiffness measurements in comparison with transient elastography. Additionally, it was proven that NAFLD patients with abnormal APRI score are highly suggestive to have abnormal pSWE. Finally, it was proved that there was not a significant correlation between pSWE and AST/ALT ratio.

\section{REFERENCES}

1. Sande, J. A., Verjee, S., Vinayak, S., Amersi, F. \& Ghesani, M. Ultrasound shear wave elastography and liver fibrosis: A Prospective Multicenter Study. World J. Hepatol. 2017; 9, 38-47.

2. Fan, Y. et al. Evolution of hepatic steatosis to fibrosis and adenoma formation in liver-specific growth hormone receptor knockout mice. Front. Endocrinol. (Lausanne). 2014; 5, 1-11.

3. To, P. \& Metabolism, L. Non-alcoholic fatty liver disease (NAFLD) perspectives to etiology, complications and lipid metabolism. 2017.

4. Sumida, Y.; Nakajima, A.; Itoh, Y. Limitations of liver biopsy and noninvasive diagnostic tests for the diagnosis of nonalcoholic fatty liver disease/nonalcoholic steatohepatitis. World J. Gastroenterol. 2014; 20, 475-485

5. Lee, J. E. et al. Noninvasive assessment of hepatic fibrosis in patients with chronic hepatitis B viral infection using magnetic resonance elastography. Korean J. Radiol. 2014; 15, 210-217.

6. Sorbi, D., Boynton, J. \& Lindor, K. D. The ratio of aspartate aminotransferase to alanine aminotransferase: Potential value in differentiating nonalcoholic steatohepatitis from alcoholic liver disease. Am. J. Gastroenterol. 1999; 94, 1018-1022.

7. Mao, Y. \& Huang, J. Commentary on: Aspartate transaminase to platelet ratio index (APRI): A simple noninvasive index assessing liver fibrosis in patients with chronic liver diseases. Hepat. Mon. 2011; 11, 376-7.

8. Ebinuma $\mathrm{H}$, Saito $\mathrm{H}$, Komuta M, Ojiro $\mathrm{K}$, Wakabayashi $\mathrm{K}$, et al. Evaluation of liver fbrosis by transient elastography using acoustic radiation force impulse: comparison with Fibroscan(®). J Gastroenterol. 2011; 46: 1238-1248. PMID: 21779759 DOI: 10.1007/s00535-011-0437-3.

9. Crespo G, Fernández-Varo G, Mariño Z, Casals G, Miquel R, Martínez SM, Gilabert R, Forns X, Jiménez W, Navasa M. ARFI, FibroScan, ELF, and their combinations in the assessment of liver fbrosis: a prospective study. J Hepatol. 2012; 57: 281-287 [PMID: 22521355 DOI: 10.1016/j.jhep.2012.03.016]

10. Ferraioli, G., Tinelli, C., Lissandrin, R., Zicchetti, M., Dal Bello, B., Filice, G., \& Filice, C. Point shear wave elastography method for assessing liver stiffness. World Journal of Gastroenterology: WJG. 2014; 20(16), 4787-4796.

11. Fraquelli, M.; Baccarin, A.; Casazza, G.; Conti, C.B.; Giunta, M.; et al. Liver stiffness measurement reliability and main determinants of point shear-wave elastography in patients with chronic liver disease. Aliment. Pharmacol. Ther. 2016, 44, 356.

12. Frulio, N. \& Trillaud, H. Ultrasound elastography in liver. Diagn. Interv. Imaging 94, 515-534 (2013).

13. Ekstedt, M. et al. Fibrosis stage is the strongest predictor for diseasespecific mortality in NAFLD after up to 33 years of follow-up. Hepatology 61, 1547-1554 (2015).

14. Nalbantoglu, I. \& Brunt, E. M. Role of liver biopsy in nonalcoholic fatty liver disease. World J. Gastroenterol. 20, 9026-9037 (2014).

15. Zheng, R.-D., Chen, Z.-R., Chen, J.-N., Lu, Y.-H. \& Chen, J. Role of Body Mass Index, Waist-to-Height and Waist-to-Hip Ratio in Prediction of Nonalcoholic Fatty Liver Disease. Gastroenterol. Res. Pract. 2012, 1-6 (2012).

16. Ferraioli, G. et al. Liver Ultrasound Elastography: An Update to the World Federation for Ultrasound in Medicine and Biology Guidelines and Recommendations. Ultrasound Med. Biol. 44, 2419-2440 (2018).

17. Sporea, I. et al. The performance of 2D SWE. GE compared to transient elastography for the evaluation of liver stiffness. Ultraschall der Medizin-European J. Ultrasound 37, SL19_3 (2016)

18. Weston, S. R. et al. Racial and ethnic distribution of nonalcoholic fatty liver in persons with newly diagnosed chronic liver disease. Hepatology 41, 372-379 (2005).

19. Benedict, M. \& Zhang, X. Non-alcoholic fatty liver disease: An expanded review. World J. Hepatol. 9, 715-732 (2017).

20. Manopriya, T., Khalid, G., Aa, A. \& Ds, S. Non-alcoholic Fatty Liver Disease ( NAFLD ) - An Emerging Public Health Problem. 5, (2016).

21. Das, S. K., Vasudevan, D. \& Balakrishnan, V. Authors' reply: Haematological parameters in patients with non-alcoholic fatty liver disease and alcoholic liver disease: Concern on findings. Singapore Med. J. 52, 383 (2011).

22. Honda, Y., Yoneda, M., Imajo, K., \& Nakajima, A. Elastography techniques for the assessment of liver fibrosis in non-alcoholic fatty liver disease. International Journal of Molecular Sciences. 2020; 21(11), 4039. doi:10.3390/ijms21114039.

23. Cassinotto, C.; Boursier, J.; de Ledinghen, V.; Lebigot, J.; Lapuyade, B.; Cales, P.; Hiriart, J.B.; Michalak, S.; Bail, B.L.; Cartier, V.; et al. Liver stiffness in nonalcoholic fatty liver disease: A comparison of supersonic shear imaging, FibroScan, and ARFI with liver biopsy. Hepatology. 2016; 63, 1817-1827.

24. Yılmaz, Y., Kanı, H. T., Demirtaş, C. Ö., Kaya, E., et al. Growing burden of nonalcoholic fatty liver disease in Turkey: A single-center experience. The Turkish Journal of Gastroenterology: The Official Journal of Turkish Society of Gastroenterology. 2019; 30(10), 892898.

25. Li, Q., Dhyani, M., Grajo, J. R., Sirlin, C. \& Samir, A. E. Current status of imaging in nonalcoholic fatty liver disease. World J. Hepatol. 10, 530-542 (2018).

26. Castera, L. Non-invasive tests for liver fibrosis in NAFLD: Creating pathways between primary healthcare and liver clinics. Liver International: Official Journal of the International Association for the Study of the Liver, 40 Suppl. 2020; 1(S1), 77-81. DOI: https://doi.org/10.1111/liv.14347. 